\title{
BLOCKS WITH A QUATERNION DEFECT GROUP OVER A 2-ADIC RING: THE CASE $\tilde{A_{4}}$
}

\author{
THORSTEN HOLM \\ Department of Pure Mathematics, University of Leeds, Leeds, LS2 9JT, U.K. \\ RADHA KESSAR and MARKUS LINCKELMANN \\ Department of Mathematical Sciences, Meston Building, Aberdeen, AB24 3UE, U.K. \\ e-mail:linckelm@maths.abdn.ac.uk
}

(Received 9 December, 2005; revised 16 October, 2006; accepted 10 November, 2006)

\begin{abstract}
Except for blocks with a cyclic or Klein four defect group, it is not known in general whether the Morita equivalence class of a block algebra over a field of prime characteristic determines that of the corresponding block algebra over a $p$ adic ring. We prove this to be the case when the defect group is quaternion of order 8 and the block algebra over an algebraically closed field $k$ of characteristic 2 is Morita equivalent to $k \tilde{A}_{4}$. The main ingredients are Erdmann's classification of tame blocks [6] and work of Cabanes and Picaronny $[4,5]$ on perfect isometries between tame blocks.
\end{abstract}

2000 Mathematics Subject Classification. 20C20.

Introduction. Throughout these notes, $\mathcal{O}$ is a complete discrete valuation ring with algebraically closed residue field $k$ of characteristic 2 and with quotient field $K$ of characteristic 0. According to Erdmann's classification in [6], if $G$ is a finite group and if $b$ is a block of $\mathcal{O} G$ having the quaternion group $Q_{8}$ of order 8 as defect group, then the block algebra $k G \bar{b}$ is Morita equivalent to either $k Q_{8}$ or $k \tilde{A}_{4}$ or the principal block algebra of $k \tilde{A}_{5}$, where here $\bar{b}$ is the canonical image of $b$ in $k G$. In the first case the block is nilpotent (cf. [3]), and it follows from Puig's structure theorem of nilpotent blocks in [8] that $\mathcal{O} G b$ is Morita equivalent to $\mathcal{O} Q_{8}$. In the remaining two cases one should expect that $\mathcal{O} G b$ is Morita equivalent to $\mathcal{O} \tilde{A}_{4}$ or the principal block algebra of $\mathcal{O} \tilde{A}_{5}$, respectively. We show this to be true in one of these two cases under the assumption that $K$ is large enough.

THEOREM A. Let $G$ be a finite group, and let b be a block of $\mathcal{O} G$ having a quaternion defect group of order 8 . Denote by $\bar{b}$ the image of $b$ in $k G$. Assume that $K G b$ is split. If $k G \bar{b}$ is Morita equivalent to $k \tilde{A}_{4}$ then $\mathcal{O} G b$ is Morita equivalent to $\mathcal{O} \tilde{A}_{4}$.

By Cabanes-Picaronny $[\mathbf{4}, \mathbf{5}]$, in the situation of Theorem A there is a perfect isometry between the character groups of $\mathcal{O} G b$ and of $\mathcal{O} \tilde{A}_{4}$. Thus Theorem A is a consequence of the following slightly more general Theorem which characterises $\mathcal{O} G b$ in terms of its center, its character group and $k \tilde{A}_{4}$; see the end of this section for more details regarding the notation.

TheOREM B. Let $A$ be an $\mathcal{O}$-free $\mathcal{O}$-algebra such that $K \otimes_{\mathcal{O}} A$ is split semi-simple and such that $k \otimes_{\mathcal{O}} A$ is Morita equivalent to $k \tilde{A}_{4}$. Assume that there is an isometry $\Phi: \mathbb{Z} \operatorname{Irr}_{K}(A) \cong \mathbb{Z} \operatorname{Irr}_{K}\left(\mathcal{O} \tilde{A}_{4}\right)$ which maps $\operatorname{Proj}(A)$ to $\operatorname{Proj}\left(\mathcal{O} \tilde{A}_{4}\right)$ such that the map sending 
$e(\chi)$ to $e(\Phi(\chi))$ for every $\chi \in \operatorname{Irr}_{K}(A)$ induces an $\mathcal{O}$-algebra isomorphism of the centers $Z(A) \cong Z\left(\mathcal{O} \tilde{A}_{4}\right)$. Then $A$ is Morita equivalent to $\mathcal{O} \tilde{A}_{4}$.

Theorem $\mathrm{B}$ is in turn a consequence of the more precise Theorem $\mathrm{C}$, describing $A$ in terms of generators and relations:

TheOREM C. Let $A$ be a basic $\mathcal{O}$-free $\mathcal{O}$-algebra such that $K \otimes_{\mathcal{O}} A$ is split semisimple and such that $k \otimes_{\mathcal{O}} A$ is isomorphic to $k \tilde{A}_{4}$. Assume that there is an isometry $\Phi: \mathbb{Z} \operatorname{Irr}_{K}(A) \cong \mathbb{Z} \operatorname{Irr}_{K}\left(\mathcal{O} \tilde{A}_{4}\right)$ which maps $\operatorname{Proj}(A)$ to $\operatorname{Proj}\left(\mathcal{O} \tilde{A}_{4}\right)$ such that the map sending $e(\chi)$ to e $(\Phi(\chi))$ for every $\chi \in \operatorname{Irr}_{K}(A)$ induces an $\mathcal{O}$-algebra isomorphism of the centers $Z(A) \cong Z\left(\mathcal{O} \tilde{A}_{4}\right)$. Then $A$ is isomorphic to the unitary $\mathcal{O}$-algebra with set of generators $\left\{e_{0}, e_{1}, e_{2}, \beta, \gamma, \delta, \eta, \lambda, \kappa\right\}$ of $A$, such that $e_{0}, e_{1}, e_{2}$ are pairwise orthogonal idempotents whose sum is 1 and satisfying the following relations:

$$
\begin{aligned}
\beta & =e_{0} \beta=\beta e_{1}, \gamma=e_{1} \gamma=\gamma e_{0} ; \\
\delta & =e_{1} \delta=\delta e_{2}, \eta=e_{2} \eta=\eta e_{1} ; \\
\lambda & =e_{2} \lambda=\lambda e_{0}, \kappa=e_{0} \kappa=\kappa e_{2} ; \\
\beta \delta & =-2 \kappa+\kappa \lambda \kappa ; \quad \eta \gamma=-2 \lambda+\lambda \kappa \lambda ; \quad \delta \lambda=-2 \gamma+\gamma \beta \gamma ; \\
\kappa \eta & =-2 \beta+\beta \gamma \beta ; \quad \lambda \beta=-2 \eta+\eta \delta \eta ; \quad \gamma \kappa=-2 \delta+\delta \eta \delta ; \\
\gamma \beta \delta & =-4 \delta+2 \delta \eta \delta ; \quad \delta \eta \gamma=-4 \gamma+2 \gamma \beta \gamma ; \lambda \kappa \eta=-4 \eta+2 \eta \delta \eta ; \\
\beta \gamma \kappa & =-4 \kappa+2 \kappa \lambda \kappa ; \quad \eta \delta \lambda=-4 \lambda+2 \lambda \kappa \lambda ; \kappa \lambda \beta=-4 \beta+2 \beta \gamma \beta ; \\
\eta \gamma \beta & =-4 \eta+2 \eta \delta \eta ; \quad \beta \delta \eta=-4 \beta+2 \beta \gamma \beta ; \delta \lambda \kappa=-4 \delta+2 \delta \eta \delta ; \\
\lambda \beta \gamma & =-4 \lambda+2 \lambda \kappa \lambda ; \kappa \eta \delta=-4 \kappa+2 \kappa \lambda \kappa ; \quad \gamma \kappa \lambda=-4 \gamma+2 \gamma \beta \gamma ; \\
\beta \delta \lambda \beta & =-8 \beta+4 \beta \gamma \beta ; \quad \delta \lambda \beta \delta=-8 \delta+4 \delta \eta \delta ; \lambda \beta \delta \lambda=-8 \lambda+4 \lambda \kappa \lambda .
\end{aligned}
$$

When reduced modulo 2, these relations seem to be more than those occuring in Erdmann's work [6] over $k$ (we recall these more precisely in $\S 2$ ); but they are not, since the extra relations over $k$ can be deduced from those given by Erdmann. We need to add in extra relations over $\mathcal{O}$ in order to ensure that the algebra we construct is $\mathcal{O}$-free of the right rank.

Since $\mathcal{O} \tilde{A}_{4}$ fulfills the hypotheses of Theorem $\mathrm{C}$ it follows that $A \cong \mathcal{O} \tilde{A}_{4}$, hence Theorem $\mathrm{C}$ indeed implies Theorem B. The proof of Theorem $\mathrm{C}$ is given at the end of Section 2.

Notation. If $A$ is an $\mathcal{O}$-algebra such that $K \otimes_{\mathcal{O}} A$ is split semi-simple, denote by $\operatorname{Irr}_{K}(A)$ the set of characters of the simple $K \otimes_{\mathcal{O}} A$-modules, viewed as central functions from $A$ to $\mathcal{O}$ and denote by $\operatorname{Irr}_{k}\left(k \otimes_{\mathcal{O}} A\right)$ the set of isomorphism classes of simple $k \otimes_{\mathcal{O}} A$-modules. We denote by $\mathbb{Z} \operatorname{Irr}_{K}(A)$ the group of characters of $A$, and by $\operatorname{Proj}(A)$ the subgroup of $\mathbb{Z} \operatorname{Irr}_{K}(A)$ generated by the characters of the projective indecomposable $A$-modules. We denote by $L^{0}(A)$ the subgroup of $\mathbb{Z} \operatorname{Irr}_{K}(A)$ of all elements which are orthogonal to $\operatorname{Proj}(A)$ with respect to the usual scalar product in $\mathbb{Z} \operatorname{Irr}_{K}(A)$. For any $\chi \in \operatorname{Irr}_{K}(A)$, we denote by $e(\chi)$ the corresponding primitive idempotent in $Z\left(K \otimes_{\mathcal{O}} A\right)$. If $A=\mathcal{O} G$ for some finite group $G$ we have the well-known formula

$$
e(\chi)=\frac{\chi(1)}{|G|} \sum_{x \in G} \chi\left(x^{-1}\right) x
$$


We refer to [1, 2] for the concept and basic properties of perfect isometries, and to [9] for general block theoretic background material.

1. Characters and perfect isometries of $\mathcal{O} \tilde{A}_{4}$. We identify $\tilde{A}_{4}=Q_{8} \rtimes C_{3}$. Let $t$ be a generator of $C_{3}$ and let $y$ be an element of order 4 in $Q_{8}$. Set $z=y^{2}$; that is, $z$ is the unique central involution of $\tilde{A}_{4}$. Then the seven elements $1, z, y, t, t^{2}, t z, t^{2} z$ are a complete set of representatives of the conjugacy classes in $\tilde{A}_{4}$.

Let $\omega$ be a primitive third root of unity in $\mathcal{O}$. The character table of $\tilde{A}_{4}$ is as follows:

$\begin{array}{rrrrrcll} & 1 & z & y & t & t^{2} & t z & t^{2} z \\ \eta_{0} & 1 & 1 & 1 & 1 & 1 & 1 & 1 \\ \eta_{1} & 1 & 1 & 1 & \omega & \omega^{2} & \omega & \omega^{2} \\ \eta_{2} & 1 & 1 & 1 & \omega^{2} & \omega & \omega^{2} & \omega \\ \eta_{3} & 3 & 3 & -1 & 0 & 0 & 0 & 0 \\ \eta_{4} & 2 & -2 & 0 & -\omega^{2} & -\omega & \omega^{2} & \omega \\ \eta_{5} & 2 & -2 & 0 & -\omega & -\omega^{2} & \omega & \omega^{2} \\ \eta_{6} & 2 & -2 & 0 & -1 & -1 & 1 & 1\end{array}$

The algebra $\mathcal{O} \tilde{A}_{4}$ has three simple modules $T_{0}, T_{1}, T_{2}$, up to isomorphism. Choosing for $T_{0}$ the trivial module and after possibly exchanging the notation for $T_{1}, T_{2}$, the ordinary decomposition matrix of $\mathcal{O} \tilde{A}_{4}$ is as follows:

$$
\left(\begin{array}{lll}
1 & 0 & 0 \\
0 & 1 & 0 \\
0 & 0 & 1 \\
1 & 1 & 1 \\
1 & 1 & 0 \\
1 & 0 & 1 \\
0 & 1 & 1
\end{array}\right)
$$

The Cartan matrix of $\mathcal{O} \tilde{A}_{4}$ is the product of the decomposition matrix with its transpose, hence equal to

$$
\left(\begin{array}{lll}
4 & 2 & 2 \\
2 & 4 & 2 \\
2 & 2 & 4
\end{array}\right) .
$$

Let $e_{0}, e_{1}, e_{2}$ be primitive idempotents in $\mathcal{O} \tilde{A}_{4}$ such that $\mathcal{O} \tilde{A}_{4} e_{i}$ is a projective cover of $T_{i}, 0 \leq i \leq 2$. By the above decomposition matrix, the characters of the projective indecomposable $\mathcal{O} \tilde{A}_{4}$-modules $\mathcal{O} \tilde{A}_{4} e_{i}$ are

$$
\begin{aligned}
& \eta_{0}+\eta_{3}+\eta_{4}+\eta_{5}, \\
& \eta_{1}+\eta_{3}+\eta_{4}+\eta_{6}, \\
& \eta_{2}+\eta_{3}+\eta_{5}+\eta_{6},
\end{aligned}
$$

respectively. Their norm is 4 , and the differences of any two different characters of projective indecomposable $\mathcal{O} \tilde{A}_{4}$-modules yields the following further elements in $\operatorname{Proj}\left(\mathcal{O} \tilde{A}_{4}\right)$ having also norm 4 :

$$
\begin{aligned}
& \eta_{0}-\eta_{1}+\eta_{5}-\eta_{6}, \\
& \eta_{0}-\eta_{2}+\eta_{4}-\eta_{6}, \\
& \eta_{1}-\eta_{2}+\eta_{4}-\eta_{5} .
\end{aligned}
$$


It is easy to check, that up to signs, these are all the elements in $\operatorname{Proj}\left(\mathcal{O} \tilde{A}_{4}\right)$ having norm 4.

A self-isometry $\Phi$ of $\mathbb{Z} \operatorname{Irr}_{K}\left(\mathcal{O} \tilde{A}_{4}\right)$ maps every $\eta_{i}$ to $\epsilon_{i} \eta_{\pi(i)}$ for some signs $\epsilon_{i} \in$ $\{1,-1\}$ and a permutation $\pi$ of $\{0,1, \ldots, 6\}$. In other words, $\Phi$ is determined by the permutation $\tau$ of the set $\{1,-1\} \times\{0,1, \ldots, 6\}$ satisfying $\tau(1, i)=\left(\epsilon_{i}, \pi(i)\right)$ and $\tau(-1, i)=\left(-\epsilon_{i}, \pi(i)\right)$ for all $i, 0 \leq i \leq 6$. If we write $i,-i$ instead of $(1, i),(-1, i)$, respectively, this becomes $\tau(i)=\epsilon_{i} \pi(i)$ and $\tau(-i)=-\epsilon_{i} \pi(i)$, with the usual cancellation rules for signs. In this way, every self-isometry $\Phi$ of $\mathbb{Z} \operatorname{Irr}_{K}\left(\mathcal{O} \tilde{A}_{4}\right)$ gets identified to a permutation of the set of symbols $\{i,-i \mid 0 \leq i \leq 6\}$. A perfect self-isometry of $\mathbb{Z} \operatorname{Irr}_{K}\left(\mathcal{O} \tilde{A}_{4}\right)$ is a self-isometry which is perfect in the sense of Broué [1]. Any such perfect self-isometry preserves $\operatorname{Proj}\left(\mathcal{O} \tilde{A}_{4}\right)$. The next Proposition implies that the converse is true, too.

Proposition 1.1. The group of all perfect self-isometries of $\mathbb{Z} \operatorname{Irr}_{K}\left(\mathcal{O} \tilde{A}_{4}\right)$ is equal to the group of all self-isometries of $\mathbb{Z} \operatorname{Irr}_{K}\left(\mathcal{O} \tilde{A}_{4}\right)$ which preserve $\operatorname{Proj}\left(\mathcal{O} \tilde{A}_{4}\right)$. This group is generated by-Id together with the set of permutations

$$
\begin{gathered}
(0,1,2)(4,6,5) \\
(1,2)(4,5) \\
(2,-3)(5,-6)
\end{gathered}
$$

Every algebra automorphism of $\mathcal{O} \tilde{A}_{4}$ induces a permutation on $\operatorname{Irr}_{K}\left(\mathcal{O} \tilde{A}_{4}\right)$ which is in fact a perfect isometry on $\mathbb{Z} \operatorname{Irr}_{K}\left(\mathcal{O} \tilde{A}_{4}\right)$. Since $\eta_{1}$ has degree 1 , it is an algebra homomorphism from $\mathcal{O} \tilde{A}_{4}$ to $\mathcal{O}$, and hence the map sending $x \in \mathcal{O} \tilde{A}_{4}$ to $\eta_{1}(x) x$ is an algebra automorphism of $\mathcal{O} \tilde{A}_{4}$ whose inverse sends $x \in \mathcal{O} \tilde{A}_{4}$ to $\eta_{2}(x) x$. The following statement is an immediate consequence from the character table of $\mathcal{O} \tilde{A}_{4}$.

LEMMA 1.2. Let $\gamma$ be the algebra automorphism of $\mathcal{O} \tilde{A}_{4}$ defined by $\gamma(x)=\eta_{1}(x) x$ for all $x \in \mathcal{O} \tilde{A}_{4}$. The permutation $\pi$ of $\{0,1, \ldots, 6\}$ defined by $\eta_{i} \circ \gamma=\eta_{\pi(i)}$ is equal to $\pi=(0,1,2)(4,6,5)$.

The anti-automorphism of $\mathcal{O} \tilde{A}_{4}$ sending $x \in \tilde{A}_{4}$ to $x^{-1}$ induces also a permutation of the set $\operatorname{Irr}_{K}\left(\mathcal{O} \tilde{A}_{4}\right)$, and this is also a perfect isometry (this holds for any finite group). This permutation can also be read off the character table.

LEMMA 1.3. Let $\iota$ be the algebra anti-automorphism of $\mathcal{O} \tilde{A}_{4}$ mapping $x \in \tilde{A}_{4}$ to $x^{-1}$. The permutation $\pi$ of $\{0,1, \ldots, 6\}$ defined by $\eta_{i} \circ \iota=\eta_{\pi(i)}$ is equal to $\pi=(1,2)(4,5)$.

Proof of 1.1. The first two permutations are perfect isometries by 1.2 and 1.3, respectively. An easy but painfully long verification shows that the bicharacter sending $(g, h) \in \tilde{A}_{4} \times \tilde{A}_{4}$ to

$$
\begin{aligned}
\eta_{0}(g) \eta_{0}(h) & +\eta_{1}(g) \eta_{1}(h)-\eta_{2}(g) \eta_{3}(h)-\eta_{3}(g) \eta_{2}(h) \\
& +\eta_{4}(g) \eta_{4}(h)-\eta_{5}(g) \eta_{6}(h)-\eta_{6}(g) \eta_{5}(h)
\end{aligned}
$$

is perfect; that is, its value at any $(g, h)$ is divisible in $\mathcal{O}$ by the orders of $C_{\tilde{A}_{4}}(g)$ and $C_{\tilde{A}_{4}}(h)$ and it vanishes if exactly one of $g, h$ has odd order. Thus the isometry given by the permutation $(2,-3)(5,-6)$ is perfect. It remains to show that these permutations, together with - Id, generate the group of all self-isometries which preserve $\operatorname{Proj}\left(\mathcal{O} \tilde{A}_{4}\right)$. 
We described above a complete list of all elements in $\operatorname{Proj}\left(\mathcal{O} \tilde{A}_{4}\right)$ having norm 4. Since the characters of the projective indecomposable modules are in that list, a selfisometry $\Phi$ of $\mathbb{Z} \operatorname{Irr}_{K}\left(\mathcal{O} \tilde{A}_{4}\right)$ preserves $\operatorname{Proj}\left(\mathcal{O} \tilde{A}_{4}\right)$ if and only if it permutes this set of norm 4 elements.

Let $\Phi$ be a self-isometry of $\mathbb{Z} \operatorname{Irr}_{K}\left(\mathcal{O} \tilde{A}_{4}\right)$ which preserves $\operatorname{Proj}\left(\mathcal{O} \tilde{A}_{4}\right)$. Then $\Phi$ preserves also the group $L^{0}\left(\mathcal{O} \tilde{A}_{4}\right)$ of generalised characters which are orthogonal to all characters in $\operatorname{Proj}\left(\mathcal{O} \tilde{A}_{4}\right)$. Up to signs, the complete list of elements in $L^{0}\left(\mathcal{O} \tilde{A}_{4}\right)$ having norm 3 is

$$
\begin{aligned}
& \eta_{0}+\eta_{1}-\eta_{4}, \eta_{0}+\eta_{2}-\eta_{5}, \eta_{0}-\eta_{3}+\eta_{6}, \\
& \eta_{1}+\eta_{2}-\eta_{6}, \eta_{1}-\eta_{3}+\eta_{5}, \eta_{2}-\eta_{3}+\eta_{4} .
\end{aligned}
$$

Up to signs again, the complete list of elements in $L^{0}\left(\mathcal{O} \tilde{A}_{4}\right)$ having norm 4 is

$$
\begin{aligned}
& \eta_{0}+\eta_{1}+\eta_{2}-\eta_{3}, \\
& \eta_{0}-\eta_{1}-\eta_{5}+\eta_{6}, \eta_{0}-\eta_{2}-\eta_{4}+\eta_{6}, \eta_{0}+\eta_{3}-\eta_{4}-\eta_{5}, \\
& \eta_{1}-\eta_{2}-\eta_{4}+\eta_{5}, \eta_{1}+\eta_{3}-\eta_{4}-\eta_{6}, \eta_{2}+\eta_{3}-\eta_{5}-\eta_{6} \text {. }
\end{aligned}
$$

The first norm 4 element in this list, $\eta_{0}+\eta_{1}+\eta_{2}-\eta_{3}$, is the only norm 4 element which is orthogonal to all other norm 4 elements in $L^{0}\left(\mathcal{O} \tilde{A}_{4}\right)$. Thus $\Phi$ has to permute the characters $\eta_{0}, \eta_{1}, \eta_{2}, \eta_{3}$ amongst each other.

Suppose first that $\Phi$ fixes $\eta_{3}$. Then, by composing $\Phi$ with a suitable product of powers of the first two permutations in the statement, we may assume that $\Phi$ fixes $\eta_{0}$, $\eta_{1}, \eta_{2}$ up to signs. By considering the first of the above norm 4 elements in $L^{0}\left(\mathcal{O} \tilde{A}_{4}\right)$ we get that $\Phi$ fixes $\eta_{0}, \eta_{1}, \eta_{2}$ all with positive signs. By considering the norm 3 elements in $L^{0}\left(\mathcal{O} \tilde{A}_{4}\right)$, it follows that $\Phi$ fixes also $\eta_{4}, \eta_{5}$ and $\eta_{6}$ with positive signs. Thus a selfisometry of $\mathbb{Z} \operatorname{Irr}_{K}\left(\mathcal{O} \tilde{A}_{4}\right)$ which preserves $\operatorname{Proj}\left(\mathcal{O} \tilde{A}_{4}\right)$ and which fixes $\eta_{3}$ is in the group generated by the set of two permutations $(0,1,2)(4,6,5)$ and $(1,2)(4,5)$.

Suppose next that $\Phi$ does not fix $\eta_{3}$. By precomposing $\Phi$ with a suitable power of $(0,1,2)(4,6,5)$ we may assume that $\Phi$ sends $\eta_{2}$ to $-\eta_{3}$. By composing $\Phi$ with a suitable power of $(0,1,2)(4,5,6)$ we may assume that $\Phi$ fixes $\eta_{0}$, up to a sign. Since $\Phi$ preserves the norm 4 element $\eta_{0}+\eta_{1}+\eta_{2}-\eta_{3}$, we necessarily have $\Phi\left(\eta_{0}\right)=\eta_{0}$. Then $\Phi$ maps $\eta_{1}$ either to $\eta_{1}$ or $\eta_{2}$ (with positive signs, again because of that same norm 4 element). In the first case, $\Phi$ fixes both $\eta_{0}, \eta_{1}$, and by checking the norm 3 elements in $L^{0}\left(\mathcal{O} \tilde{A}_{4}\right)$ one gets $\Phi=(2,-3)(5,-6)$. In the second case, again checking on norm 3 elements, one gets $\Phi=(1,2,-3)(4,5,-6)$, but this is already the product of $(1,2)(4,5)$ and $(2,-3)(5,-6)$.

2. The algebra $A$. Let $A$ be a basic $\mathcal{O}$-algebra fulfilling the hypotheses of Theorem $\mathrm{B}$; that is, $K \otimes_{\mathcal{O}} A$ is split semi-simple, $k \otimes_{\mathcal{O}} A$ is isomorphic to $k \tilde{A}_{4}$, and there is an isometry $\mathbb{Z} \operatorname{Irr}_{K}(A) \cong \mathbb{Z} \operatorname{Irr}_{K}\left(\mathcal{O} \tilde{A}_{4}\right)$ mapping $\operatorname{Proj}(A)$ to $\operatorname{Proj}\left(\mathcal{O} \tilde{A}_{4}\right)$ and inducing an isomorphism $Z(A) \cong Z\left(\mathcal{O} \tilde{A}_{4}\right)$. There is a "compatible choice" for these isomorphisms.

Proposition 2.1. There is an algebra isomorphism $\alpha: k \otimes_{\mathcal{O}} A \cong k \tilde{A}_{4}$ and an isometry $\Phi: \mathbb{Z} \operatorname{Irr}_{K}(A) \cong \mathbb{Z}_{\operatorname{Irr}_{K}}\left(\mathcal{O} \tilde{A}_{4}\right)$ mapping $\operatorname{Proj}(A)$ to $\operatorname{Proj}\left(\mathcal{O} \tilde{A}_{4}\right)$ with the following properties:

(i) $\Phi \operatorname{maps}_{\operatorname{Irr}_{K}}(A)$ onto $\operatorname{Irr}_{K}\left(\mathcal{O} \tilde{A}_{4}\right)$; that is, all signs are +1 .

(ii) The map sending $e(\chi)$ to $e(\Phi(\chi))$ for every $\chi \in \operatorname{Irr}_{K}(A)$ induces an isomorphism $Z(A) \cong Z\left(\mathcal{O} \tilde{A}_{4}\right)$. 
(iii) For any primitive idempotents $e \in A$ and $f \in \mathcal{O} \tilde{A}_{4}$ and every $\chi \in \operatorname{Irr}_{K}(A)$ such that $\alpha(\bar{e})=\bar{f}$ we have $\chi(e)=\Phi(\chi)(f)$; that is, $A$ and $\mathcal{O} \tilde{A}_{4}$ have the same decomposition matrices through $\alpha$ and $\Phi$.

Proof. The $\mathcal{O}$-rank of $A$ is 24 and also the sum of the squares of the degrees of the seven irreducible $K$-linear characters of $A$; thus every irreducible character of $A$ has degree smaller than 5 . Also, there is no character of degree 4 because $24-4^{2}=8$ cannot be written as a sum of six squares of the degrees of the six remaining characters. But there must be a character of degree 3; if not, 24 would be the sum of seven squares all either 1 or 4 , which is not possible. Thus the squares of the degrees of the six remaining characters add up to $24-3^{2}=15$, and the only way to do this is with three characters of degree 1 and three characters of degree 2.

This proves that the character degrees of the irreducible characters of $A$ and of $\mathcal{O} \tilde{A}_{4}$ coincide for some bijection $\operatorname{Irr}_{K}(A) \cong \operatorname{Irr}_{K}\left(\mathcal{O} \tilde{A}_{4}\right)$. Since the decomposition matrix of $A$ multiplied with its transpose yields the Cartan matrix of $A$-which is equal to that of $k \tilde{A}_{4}$ - the algebra $A$ has in fact the same decomposition matrix as $\mathcal{O} \tilde{A}_{4}$ for a suitable bijection $\Phi: \operatorname{Irr}_{K}(A) \cong \operatorname{Irr}_{K}\left(\mathcal{O} \tilde{A}_{4}\right)$ and the bijection $\operatorname{Irr}_{k}\left(k \otimes_{\mathcal{O}} A\right) \cong \operatorname{Irr}_{k}\left(k \tilde{A}_{4}\right)$ induced by $\alpha$. Extend $\Phi$ to a $\mathbb{Z}$-linear isomorphism $\mathbb{Z} \operatorname{Irr}_{K}(A) \cong \mathbb{Z} \operatorname{Irr}_{K}\left(\mathcal{O} \tilde{A}_{4}\right)$, still denoted by $\Phi$. By construction, $\Phi$ sends the characters of the projective indecomposable $A$-modules to the characters of the projective indecomposable $\mathcal{O} \tilde{A}_{4}$-modules; in particular, $\Phi$ maps $\operatorname{Proj}(A)$ to $\operatorname{Proj}\left(\mathcal{O} \tilde{A}_{4}\right)$. It remains to see that the map sending $e(\chi)$ to $e(\Phi(\chi))$ for every $\chi \in \operatorname{Irr}_{K}(A)$ induces an isomorphism $Z(A) \cong Z\left(\mathcal{O} \tilde{A}_{4}\right)$. For any $i, 0 \leq i \leq 6$, denote by $\chi_{i}$ the irreducible character of $A$ such that $\Phi\left(\chi_{i}\right)=\eta_{i}$. As in the proof of 1.1, we have a distinguished norm 4 element in $L^{0}(A)$ which is orthogonal to all other norm 4 elements in $L^{0}(A)$, namely $\chi_{0}+\chi_{1}+\chi_{2}-\chi_{3}$. Thus, if $\Psi: \mathbb{Z} \operatorname{Irr}_{K}(A) \cong \mathbb{Z} \operatorname{Irr}_{K}\left(\mathcal{O} \tilde{A}_{4}\right)$ is some isometry mapping $\operatorname{Proj}(A)$ to $\operatorname{Proj}\left(\mathcal{O} \tilde{A}_{4}\right)$ and inducing an isomorphism $Z(A) \cong$ $Z\left(\mathcal{O} \tilde{A}_{4}\right)$, then $\Psi\left(\chi_{0}+\chi_{1}+\chi_{2}-\chi_{3}\right)= \pm\left(\eta_{0}+\eta_{1}+\eta_{2}-\eta_{3}\right)$. By Proposition 1.1, there is a perfect self-isometry $\mu$ of $\mathbb{Z} \operatorname{Irr}_{K}\left(\mathcal{O} \tilde{A}_{4}\right)$ such that $\Phi=\mu \circ \Psi$.

REMARK 2.2. If we assume that $A$ is Morita equivalent to some block algebra with $Q_{8}$ as defect group, then Proposition 2.1 follows also from the work of Cabanes and Picaronny in $[\mathbf{4}, \mathbf{5}]$.

Since $k \otimes_{\mathcal{O}} A \cong k \tilde{A}_{4}$, the quiver of $A$ is the same as that of $k \tilde{A}_{4}$, thus of the following form:

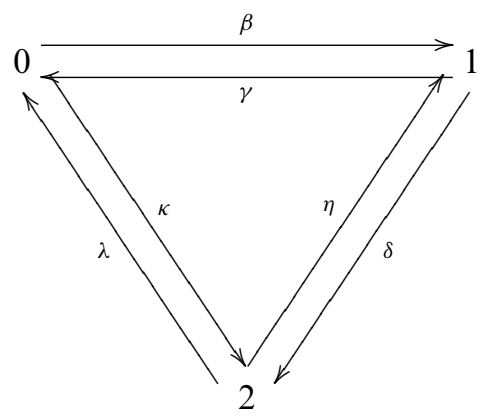


Write $\bar{a}$ for the image of $a \in A$ in $\bar{A}=k \otimes_{\mathcal{O}} A \cong k \tilde{A}_{4}$. The generators $\beta, \gamma, \delta, \kappa, \lambda$, $\eta$ can be chosen such that their images in $\bar{A}$ fulfill the following relations:

$$
\begin{aligned}
& \bar{\beta} \bar{\delta}=\bar{\kappa} \bar{\lambda} \bar{\kappa}, \\
& \bar{\eta} \bar{\gamma}=\bar{\lambda} \bar{\kappa} \bar{\lambda}, \\
& \bar{\delta} \bar{\lambda}=\bar{\gamma} \bar{\beta} \bar{\gamma} \\
& \bar{\kappa} \bar{\eta}=\bar{\beta} \bar{\gamma} \bar{\beta}, \\
& \bar{\lambda} \bar{\beta}=\bar{\eta} \bar{\delta} \bar{\eta}, \\
& \bar{\gamma} \bar{\kappa}=\bar{\delta} \bar{\eta} \bar{\delta}
\end{aligned}
$$

and

$$
\bar{\gamma} \bar{\beta} \bar{\delta}=\bar{\delta} \bar{\eta} \bar{\gamma}=\bar{\lambda} \bar{\kappa} \bar{\eta}=0 .
$$

In order to determine the algebra structure of $A$, we have to "lift" these relations over $\mathcal{O}$.

We fix an algebra isomorphism $\alpha: k \otimes_{\mathcal{O}} A \cong k \tilde{A}_{4}$ and an isometry $\Phi: \mathbb{Z}_{\operatorname{Irr}_{K}}(A) \cong$ $\mathbb{Z} \operatorname{Irr}_{K}\left(\mathcal{O} \tilde{A}_{4}\right)$ satisfying the conclusions of Proposition 2.1 . We denote by $\chi_{i}$ the unique irreducible $K$-linear character of $A$ such that $\Phi\left(\chi_{i}\right)=\eta_{i}$ for all $i, 0 \leq i \leq 6$.

The characters $\eta_{0}, \eta_{1}, \eta_{2}, \eta_{3}$ of $\mathcal{O} \tilde{A}_{4}$ have height zero, the characters $\eta_{4}, \eta_{5}, \eta_{6}$ have height one. Thus, via the isomorphism of the centers induced by $\Phi$, it follows that for $0 \leq i \leq 3$ we have $8 e\left(\chi_{i}\right) \in A$, and for $4 \leq j \leq 6$ we have $4 e\left(\chi_{j}\right) \in A$. We can in fact describe an $\mathcal{O}$-basis of $Z(A)$ in terms of the centrally primitive idempotents $e\left(\chi_{i}\right)$. The strategy is now to play off the descriptions of $Z\left(k \otimes_{\mathcal{O}} A\right)$ in terms of the generators in the quiver and of $Z(A)$ in terms of the centrally primitive idempotents $e\left(\chi_{i}\right)$.

LEMMA 2.3. The following elements of $Z\left(K \otimes_{\mathcal{O}} A\right)$ are all contained in the radical $J(Z(A))$ :

$$
\begin{aligned}
s & =2 e\left(\chi_{4}\right)+2 e\left(\chi_{5}\right)+2 e\left(\chi_{6}\right), \\
z_{0} & =4 e\left(\chi_{2}\right)+4 e\left(\chi_{3}\right)+2 e\left(\chi_{4}\right), \\
z_{1} & =4 e\left(\chi_{1}\right)+4 e\left(\chi_{3}\right)+2 e\left(\chi_{5}\right), \\
z_{2} & =4 e\left(\chi_{0}\right)+4 e\left(\chi_{3}\right)+2 e\left(\chi_{6}\right), \\
y_{0} & =4 e\left(\chi_{1}\right)+4 e\left(\chi_{2}\right)+2 e\left(\chi_{4}\right)+2 e\left(\chi_{5}\right), \\
y_{1} & =4 e\left(\chi_{0}\right)+4 e\left(\chi_{2}\right)+2 e\left(\chi_{4}\right)+2 e\left(\chi_{6}\right), \\
y_{2} & =4 e\left(\chi_{0}\right)+4 e\left(\chi_{1}\right)+2 e\left(\chi_{5}\right)+2 e\left(\chi_{6}\right) .
\end{aligned}
$$

Moreover, for any two different $i, j$ in $\{0,1,2\}$ the set

$$
\left\{1, z_{i}, z_{j}, s, 8 e\left(\chi_{3}\right), 4 e\left(\chi_{i+4}\right), 4 e\left(\chi_{j+4}\right)\right\}
$$

is an $\mathcal{O}$-basis of $Z(A)$.

Proof. In view of Proposition 2.1 we may assume that $A=\mathcal{O} \tilde{A}_{4}$ and that $\chi_{i}=\eta_{i}$ for $0 \leq i \leq 6$. This is just an explicit verification, using the character table of $\tilde{A}_{4}$. One verifies first that $z_{0} \in A$. By symmetry, this implies that $z_{1}, z_{2}$ are also in $A$. Then $y_{0}=z_{0}+z_{1}-8 e\left(\chi_{3}\right)$ is in $A$, similarly for the $y_{1}, y_{2}$. An equally easy computation shows that $s \in A$. Thus all the given elements belong to $Z(A)$. None of these elements is invertible, so they all belong to $J(Z(A))$ because $Z(A)$ is local. 
In order to see the last statement on the basis of $Z(A)$, we may assume that $i=0$ and $j=1$. For any $x \in \tilde{A}_{4}$ denote by $\underline{x}$ the conjugacy class sum of $x$ in $\mathcal{O} \tilde{A}_{4}$. The orthogonality relations imply the well-known formula

$$
\underline{x}=\sum_{0 \leq m \leq 6} \frac{\chi_{m}\left(\underline{x}^{-1}\right)}{\chi_{m}(1)} e\left(\chi_{m}\right) .
$$

Thus, for the seven conjugacy classes in $\tilde{A}_{4}$, we have

$$
\begin{aligned}
\underline{1} & =e\left(\chi_{0}\right)+e\left(\chi_{1}\right)+e\left(\chi_{2}\right)+e\left(\chi_{3}\right)+e\left(\chi_{4}\right)+e\left(\chi_{5}\right)+e\left(\chi_{6}\right) ; \\
\underline{z} & =e\left(\chi_{0}\right)+e\left(\chi_{1}\right)+e\left(\chi_{2}\right)+e\left(\chi_{3}\right)-e\left(\chi_{4}\right)-e\left(\chi_{5}\right)-e\left(\chi_{6}\right) ; \\
\underline{y} & =6 e\left(\chi_{0}\right)+6 e\left(\chi_{1}\right)+6 e\left(\chi_{2}\right)-2 e\left(\chi_{3}\right) ; \\
\underline{t} & =4 e\left(\chi_{0}\right)+4 \omega^{2} e\left(\chi_{1}\right)+4 \omega e\left(\chi_{2}\right)-2 \omega e\left(\chi_{4}\right)-2 \omega^{2} e\left(\chi_{5}\right)-2 e\left(\chi_{6}\right) ; \\
\underline{t}^{2} & =4 e\left(\chi_{0}\right)+4 \omega e\left(\chi_{1}\right)+4 \omega^{2} e\left(\chi_{2}\right)-2 \omega^{2} e\left(\chi_{4}\right)-2 \omega e\left(\chi_{5}\right)-2 e\left(\chi_{6}\right) ; \\
\underline{t z} & =4 e\left(\chi_{0}\right)+4 \omega^{2} e\left(\chi_{1}\right)+4 \omega e\left(\chi_{2}\right)+2 \omega e\left(\chi_{4}\right)+2 \omega^{2} e\left(\chi_{5}\right)+2 e\left(\chi_{6}\right) ; \\
\underline{t^{2} z} & =4 e\left(\chi_{0}\right)+4 \omega e\left(\chi_{1}\right)+4 \omega^{2} e\left(\chi_{2}\right)+2 \omega^{2} e\left(\chi_{4}\right)+2 \omega e\left(\chi_{5}\right)+2 e\left(\chi_{6}\right) .
\end{aligned}
$$

We show that they are all in the $\mathcal{O}$-linear span of the elements in the set

$$
\left\{1, z_{0}, z_{1}, s, 8 e\left(\chi_{3}\right), 4 e\left(\chi_{4}\right), 4 e\left(\chi_{5}\right)\right\} .
$$

Note first that

$$
\begin{aligned}
z_{2} & =4 \cdot 1-z_{0}-z_{1}-s+8 e\left(\chi_{3}\right), \\
4 e\left(\chi_{6}\right) & =2 s-4 e\left(\chi_{4}\right)-4 e\left(\chi_{5}\right)
\end{aligned}
$$

are in the $\mathcal{O}$-linear span of this set. One easily verifies now that

$$
\begin{aligned}
\underline{z} & =1-s, \\
\underline{y} & =6 \cdot 1-3 s-8 e\left(\chi_{3}\right), \\
\underline{t} & =\omega z_{0}+\omega^{2} z_{1}+z_{2}-4 \omega e\left(\chi_{4}\right)-4 \omega^{2} e\left(\chi_{5}\right)-4 e\left(\chi_{6}\right), \\
\underline{t^{2}} & =\omega^{2} z_{0}+\omega z_{1}+z_{2}-4 \omega^{2} e\left(\chi_{4}\right)-4 \omega e\left(\chi_{5}\right)-4 e\left(\chi_{6}\right), \\
\underline{t z} & =\omega z_{0}+\omega^{2} z_{1}+z_{2}, \\
\underline{t^{2} z} & =\omega^{2} z_{0}+\omega z_{1}+z_{2} .
\end{aligned}
$$

This concludes the proof of 2.3.

The center of $\bar{A}=k \otimes_{\mathcal{O}} A$ can easily be described in terms of the generators in the quiver of $A$ :

LEMMA 2.4. The following set is a k-basis of $Z(\bar{A})$.

$$
\{1, \bar{\beta} \bar{\gamma}+\bar{\gamma} \bar{\beta}, \bar{\kappa} \bar{\lambda}+\bar{\lambda} \bar{\kappa}, \bar{\eta} \bar{\delta}+\bar{\delta} \bar{\eta}, \bar{\beta} \bar{\delta} \bar{\lambda}, \bar{\delta} \bar{\lambda} \bar{\beta}, \bar{\lambda} \bar{\beta} \bar{\delta}\}
$$

Proof. Straightforward verification, using $(\bar{\beta} \bar{\gamma})^{2}=\bar{\beta} \bar{\delta} \bar{\lambda}$ and the similar relations for the other elements in the given set.

Proposition 2.5. For any primitive idempotent $e$ in $A$ we have $Z(A) e=e A e$. Moreover, 
(i) the set $\left\{e_{0}, z_{0} e_{0}, z_{1} e_{0}, 4 e\left(\chi_{4}\right) e_{0}\right\}$ is an $\mathcal{O}$-basis of $e_{0} A e_{0}$.

(ii) the set $\left\{e_{1}, z_{0} e_{1}, z_{2} e_{1}, 4 e\left(\chi_{4}\right) e_{1}\right\}$ is an $\mathcal{O}$-basis of $e_{1} A e_{1}$;

(iii) the set $\left\{e_{2}, z_{1} e_{2}, z_{2} e_{2}, 4 e\left(\chi_{5}\right) e_{2}\right\}$ is an $\mathcal{O}$-basis of $e_{2} A e_{2}$.

Proof. Since $Z(A) \cong Z\left(\mathcal{O} \tilde{A}_{4}\right)$ and $Z(\bar{A}) \cong Z\left(k \tilde{A}_{4}\right)$, the canonical map $A \rightarrow \bar{A}$ maps $Z(A)$ onto $Z(\bar{A})$ and hence $Z(A) e$ onto $Z(\bar{A}) \bar{e}$. By Nakayama's Lemma, it suffices to show that $Z(\bar{A}) \bar{e}=\bar{e} \bar{A} \bar{e}$. Now $\operatorname{dim}_{k}(\bar{e} \bar{A} \bar{e})=4$ by the Cartan matrix, and so we have only to show that $\operatorname{dim}_{k}(Z(\bar{A}) \bar{e})=4$. By the symmetry of the quiver of $A$, we may assume that $e$ corresponds to the vertex labelled 0 . Then the set $\{\bar{e}, \bar{\beta} \bar{\gamma}, \bar{\kappa} \bar{\lambda}, \bar{\beta} \bar{\delta} \bar{\lambda}\}$ is a $k$-basis of $Z(\bar{A}) \bar{e}$ by 2.4 ; in particular, $\operatorname{dim}_{k}(Z(\bar{A}) \bar{e})=4$ as required. This shows that $e A e=Z(A) e$.

In order to prove (i), note that the set

$$
\left\{e_{0}, z_{0} e_{0} z_{1} e_{0}, s e_{0}, 8 e\left(\chi_{3}\right) e_{0}, 4 e\left(\chi_{4}\right) e_{0}, 4 e\left(\chi_{5}\right) e_{0}\right\}
$$

generates $e_{0} A e_{0}$ as $\mathcal{O}$-module, by the first statement and by the $\mathcal{O}$-basis of $Z(A)$ described in 2.3. Now we have

$$
\begin{aligned}
8 e\left(\chi_{3}\right) e_{0} & =2 z_{0} e_{0}-4 e\left(\chi_{4}\right) e_{0}, \\
4 e\left(\chi_{5}\right) e_{0} & =2 z_{0} e_{0}-2 z_{1} e_{0}+4 e\left(\chi_{4}\right) e_{0}, \\
s e_{0} & =\left(z_{1}-z_{0}+4 e\left(\chi_{4}\right)\right) e_{0} .
\end{aligned}
$$

Thus the set given in (i) generates $e_{0} A e_{0}$ as $\mathcal{O}$-module, and hence is a basis since the $\mathcal{O}$-rank of $e_{0} A e_{0}$ is 4 . The same arguments show (ii) and (iii).

Proposition 2.6. We can choose the generators $\beta, \gamma, \delta, \eta, \lambda, \kappa$ in such a way that

(i) $A \gamma$ is the unique $\mathcal{O}$-pure submodule of $A e_{0}$ with character $\chi_{3}+\chi_{4}$;

(ii) $A \lambda$ is the unique $\mathcal{O}$-pure submodule of $A e_{0}$ with character $\chi_{3}+\chi_{5}$;

(iii) $A \eta$ is the unique $\mathcal{O}$-pure submodule of $A e_{1}$ with character $\chi_{3}+\chi_{6}$;

(iv) $A \beta$ is the unique $\mathcal{O}$-pure submodule of $A e_{1}$ with character $\chi_{3}+\chi_{4}$;

(v) $A \kappa$ is the unique $\mathcal{O}$-pure submodule of $A e_{2}$ with character $\chi_{3}+\chi_{5}$;

(vi) $A \delta$ is the unique $\mathcal{O}$-pure submodule of $A e_{2}$ with character $\chi_{3}+\chi_{6}$.

Proof. We are going to prove (i); by the symmetry of the quiver of $A$ one gets all other statements. Observe first that $\bar{A} \bar{\gamma}$ is the unique 5-dimensional submodule of $A e_{0}$ with composition factors $2\left[S_{0}\right], 2\left[S_{1}\right]$, [S $\left.S_{2}\right]$. Indeed, the set $\{\bar{\gamma}, \bar{\beta} \bar{\gamma}, \bar{\eta} \bar{\gamma}, \bar{\gamma} \bar{\beta} \bar{\gamma}, \bar{\beta} \bar{\gamma} \bar{\beta} \bar{\gamma}\}$ is a $k$-basis of $\bar{A} \bar{\gamma}$, and we have $\bar{\gamma}, \bar{\gamma} \bar{\beta} \bar{\gamma} \in \bar{e}_{0} \bar{A} \bar{e}_{0}$, yielding the two composition factors isomorphic to $S_{0}$, we have $\bar{\beta} \bar{\gamma}, \bar{\beta} \bar{\gamma} \bar{\beta} \bar{\gamma} \in \bar{e}_{1} \bar{A} \bar{e}_{0}$, yielding the two composition factors isomorphic to $S_{1}$, and finally $\bar{\eta} \bar{\gamma} \in \bar{e}_{2} \bar{A} \bar{e}_{0}$, yielding the remaining composition factor isomorphic to $S_{2}$. One checks that there is no other submodule with exactly these composition factors. Now there is exactly one $\mathcal{O}$-pure submodule $U$ of $A e_{0}$ whose reduction modulo $J(\mathcal{O})$ has composition series $2\left[S_{0}\right]+2\left[S_{1}\right]+\left[S_{2}\right]$, namely the unique $\mathcal{O}$-pure submodule of $A e_{0}$ with character $\chi_{3}+\chi_{4}$; this is a direct consequence of the decomposition matrix. One constructs $U$ as follows: write $K \otimes_{\mathcal{O}} A e_{0}=X_{0} \oplus X_{3} \oplus X_{4} \oplus$ $X_{5}$, where $X_{j}$ is the unique submodule of $K \otimes_{\mathcal{O}} A e_{0}$ with character $\chi_{j}$ for $j \in\{0,3,4,5\}$, and then $U=A e_{0} \cap\left(X_{3} \oplus X_{4}\right)$. Take now for $\gamma$ any inverse image in $U$ of $\bar{\gamma}$. Then $A \gamma \subseteq U$ and $U \subseteq A \gamma+J(\mathcal{O}) U$. Thus $A \gamma=U$ by Nakayama's Lemma.

COROLLARY 2.7. If the generators $\beta, \gamma, \delta \eta, \lambda, \kappa$ are chosen such that they fulfill the conclusions of 2.6 then, with the notation of 2.3, the following hold.

(i) $y_{0} \delta=y_{0} \eta=0$. 
(ii) $y_{1} \lambda=y_{1} \kappa=0$.

(iii) $y_{2} \gamma=y_{2} \beta=0$. hold:

Proposition 2.8. We can choose the generators $\beta, \gamma, \delta, \eta, \lambda, \kappa$ such that the following

$$
\begin{aligned}
\beta \gamma & =z_{0} e_{0}=4 e\left(\chi_{3}\right) e_{0}+2 e\left(\chi_{4}\right) e_{0} ; \\
\gamma \beta & =z_{0} e_{1}=4 e\left(\chi_{3}\right) e_{1}+2 e\left(\chi_{4}\right) e_{1} ; \\
\delta \eta & =z_{2} e_{1}=4 e\left(\chi_{3}\right) e_{1}+2 e\left(\chi_{6}\right) e_{1} ; \\
\eta \delta & =z_{2} e_{2}=4 e\left(\chi_{3}\right) e_{2}+2 e\left(\chi_{6}\right) e_{2} ; \\
\lambda \kappa & =z_{1} e_{2}=4 e\left(\chi_{3}\right) e_{2}+2 e\left(\chi_{5}\right) e_{2} ; \\
\kappa \lambda & =z_{1} e_{0}=4 e\left(\chi_{3}\right) e_{0}+2 e\left(\chi_{5}\right) e_{0} ; \\
\beta \delta \lambda & =\kappa \eta \gamma=8 e\left(\chi_{3}\right) e_{0} ; \\
\delta \lambda \beta & =\gamma \kappa \eta=8 e\left(\chi_{3}\right) e_{1} ; \\
\lambda \beta \delta & =\eta \gamma \kappa=8 e\left(\chi_{3}\right) e_{2} .
\end{aligned}
$$

Proof. In view of the decomposition matrix of $A$ we have $e_{0}=e\left(\chi_{0}\right) e_{0}+e\left(\chi_{3}\right) e_{0}+$ $e\left(\chi_{4}\right) e_{0}+e\left(\chi_{5}\right) e_{0}$. Moreover, the elements $e\left(\chi_{0}\right) e_{0}, e\left(\chi_{3}\right) e_{0}, e\left(\chi_{4}\right) e_{0}, e\left(\chi_{5}\right) e_{0}$ are $K$ linearly independent because they are pairwise orthogonal idempotents in $K \otimes_{\mathcal{O}} A$. Similar statements hold for $e_{1}, e_{2}$.

We assume a choice of generators fulfilling 2.6. We have $A \beta \gamma \subseteq A \gamma$, and the submodule $A \gamma$ of $A e_{0}$ has character $\chi_{3}+\chi_{4}$ by 2.6. Thus $\beta \gamma$ is a $K$-linear combination of $e\left(\chi_{3}\right) e_{1}$ and $e\left(\chi_{4}\right) e_{1}$. But also $\beta \gamma$ is an $\mathcal{O}$-linear combination of the basis elements $e_{0}, z_{0} e_{0} z_{1} e_{0}, 4 e\left(\chi_{4}\right) e_{0}$ given in 2.5 in which none of $\chi_{1}, \chi_{5}$ shows up. Therefore $\beta \gamma$ is in fact an $\mathcal{O}$-linear combination of the elements $z_{0} e_{0}, 4 e\left(\chi_{4}\right) e_{0}$; say

$$
\beta \gamma=\left(\mu_{0} z_{0} e_{0}+4 v_{0} e\left(\chi_{4}\right)\right) e_{0}=\left(4 \mu_{0} e\left(\chi_{3}\right)+2\left(\mu_{0}+2 v_{0}\right) e\left(\chi_{4}\right)\right) e_{0}
$$

for some coefficients $\mu_{0}, v_{0} \in \mathcal{O}$. Hence

$$
(\beta \gamma)^{2}=\left(16 \mu_{0}^{2} e\left(\chi_{3}\right)+4\left(\mu_{0}+2 \nu_{0}\right)^{2} e\left(\chi_{4}\right)\right) e_{0} .
$$

Now $(\bar{\beta} \bar{\gamma})^{2} \neq 0$, and therefore $\mu_{0} \in \mathcal{O}^{\times}$. Set now

$$
a_{0}=1+v_{0} \mu_{0}^{-1} y_{0} .
$$

Since $y_{0} \in J(Z(A))$ by 2.3 we have $a_{0} \in Z(A)^{\times}$. A trivial verification, comparing coefficients, shows that we have

$$
\beta \gamma=\mu_{0} z_{0} a_{0} e_{0}
$$

Since $\gamma=e_{1} \gamma=\gamma e_{0}$, multiplying this with $\gamma$ on the left yields

$$
\gamma \beta \gamma=\mu_{0} z_{0} a_{0} e_{1} \gamma
$$

Now both $\gamma \beta$ and $\mu_{0} z_{0} a_{0} e_{1}$ are contained in the pure submodule $A \beta$ of $A e_{1}$ with character $\chi_{3}+\chi_{4}$, by 2.6 and the nature of the element $z_{0}$. Right multiplication by $\gamma$ on this submodule is therefore injective (the annihilator of $\gamma$ in $A e_{1}$ is the pure submodule 
with character $\chi_{1}+\chi_{6}$ ). Hence the previous equality implies also the equality

$$
\gamma \beta=\mu_{0} z_{0} a_{0} e_{1}
$$

In an entirely analogous way one finds scalars $\mu_{1}, \mu_{2} \in \mathcal{O}^{\times}$such that, setting $a_{1}=$ $1+v_{1} \mu_{1}^{-1} y_{1}$ and $a_{2}=1+v_{2} \mu_{2}^{-1} y_{2}$, one gets the equalities

$$
\begin{gathered}
\delta \eta=\mu_{2} z_{2} a_{2} e_{1}, \eta \delta=\mu_{2} z_{2} a_{2} e_{2}, \\
\lambda \kappa=\mu_{1} z_{1} a_{1} e_{2}, \kappa \lambda=\mu_{1} z_{1} a_{1} e_{0} .
\end{gathered}
$$

Moreover, the equalities in 2.7 imply the following equalities:

$$
\begin{aligned}
& a_{0} \delta=\delta, a_{0} \eta=\eta, \\
& a_{1} \lambda=\lambda, a_{1} \kappa=\kappa \\
& a_{2} \gamma=\gamma, a_{2} \beta=\beta
\end{aligned}
$$

If we replace now $\beta$ by $a_{0}^{-1} \beta$, this is not going to change the properties stated in 2.6 and also this is not changing the relations over $k$ of the quiver. Similarly, we can replace $\delta$ by $a_{2}^{-1} \delta$ and $\lambda$ by $a_{1}^{-1} \lambda$. Then the generators $\beta, \gamma, \delta, \eta, \lambda, \kappa$ still fulfill 2.6 , and in addition, we have now the following equalities:

$$
\begin{aligned}
\beta \gamma & =\mu_{0} z_{0} e_{0}, \quad \gamma \beta=\mu_{0} z_{0} e_{1}, \\
\delta \eta & =\mu_{2} z_{2} e_{1}, \eta \delta=\mu_{2} z_{2} e_{2}, \\
\lambda \kappa & =\mu_{1} z_{1} e_{2}, \kappa \lambda=\mu_{1} z_{1} e_{0} .
\end{aligned}
$$

We have to get rid of the scalars $\mu_{0}, \mu_{1}, \mu_{2}$. Since $\chi_{3}$ is the only character appearing in the characters of all projective indecomposable $A$-modules we have

$$
\beta \delta \lambda=8 \mu e\left(\chi_{3}\right) e_{0}
$$

for some $\mu \in \mathcal{O}$. Then actually $\mu \in \mathcal{O}^{\times}$because $\bar{\beta} \bar{\delta} \bar{\lambda} \neq 0$. Moreover, $\beta \delta \lambda \beta=8 \mu e\left(\chi_{3}\right) \beta$, and hence also

$$
\delta \lambda \beta=8 \mu e\left(\chi_{3}\right) e_{1} .
$$

The same argument applied again yields

$$
\lambda \beta \delta=8 \mu e\left(\chi_{3}\right) e_{2} .
$$

Applying this argument to the arrows in the quiver in the opposite direction implies that there is $\mu^{\prime} \in \mathcal{O}^{\times}$such that

$$
\begin{aligned}
& \kappa \eta \gamma=8 \mu^{\prime} e\left(\chi_{3}\right) e_{0}, \\
& \eta \gamma \kappa=8 \mu^{\prime} e\left(\chi_{3}\right) e_{2}, \\
& \gamma \kappa \eta=8 \mu^{\prime} e\left(\chi_{3}\right) e_{1} .
\end{aligned}
$$

Now $\bar{\beta} \bar{\delta} \bar{\lambda}=\bar{\kappa} \bar{\lambda} \bar{\kappa} \bar{\lambda}=\bar{\kappa} \bar{\eta} \bar{\gamma}$, and hence $\mu^{\prime}=\mu(1+v)$ for some $v \in J(\mathcal{O})$. Note that we can always multiply any of the generators by any scalar in $1+J(\mathcal{O})$ without modifying the relations over $k$. Thus, if we replace $\kappa$ by $(1+v) \kappa$, we may assume that $\mu^{\prime}=\mu$. 
Since the set $\{\kappa, \kappa \lambda \kappa\}$ is an $\mathcal{O}$-basis of $e_{0} A e_{2}$, we can write

$$
\beta \delta=a \kappa+b \kappa \lambda \kappa
$$

for some unique scalars $a, b \in \mathcal{O}$. Multiplying this by $\lambda$ yields

$$
8 \mu e\left(\chi_{3}\right) e_{0}=\beta \delta \lambda=a \kappa \lambda+b(\kappa \lambda)^{2}=\left(a \mu_{1} z_{1}+b \mu_{1}^{2} z_{1}^{2}\right) e_{0} .
$$

By comparing the coefficients at $e\left(\chi_{3}\right) e_{0}$ and $e\left(\chi_{5}\right) e_{0}$ of the left and right expression in this equality, we get the equations

$$
\begin{aligned}
8 \mu & =4 a \mu_{1}+16 b \mu_{1}^{2}, \\
0 & =2 a \mu_{1}+4 b \mu_{1}^{2} .
\end{aligned}
$$

An easy computation shows that $b=\frac{\mu}{\mu_{1}^{2}}$. Moreover, since $\bar{\beta} \bar{\delta} \bar{\lambda}=(\bar{\kappa} \bar{\lambda})^{2}$ we have $\bar{a}=0$ and $\bar{b}=1_{k}$, hence $b=\frac{\mu}{\mu_{1}^{2}} \in 1+J(\mathcal{O})$. By repeating the same argument we find also that the coefficients $\frac{\mu}{\mu_{0}^{2}}, \frac{\mu}{\mu_{2}^{2}}$ are in $1+J(\mathcal{O})$.

Next, we compute $\beta \delta \lambda \kappa \eta \gamma$ in two different ways: on one hand we have

$$
(\beta \delta \lambda)(\kappa \eta \gamma)=64 \mu^{2} e\left(\chi_{3}\right) e_{0},
$$

and on the other hand we have

$$
\beta(\delta(\lambda \kappa) \eta) \gamma=\mu_{0} \mu_{1} \mu_{2} z_{0} z_{1} z_{2} e\left(\chi_{3}\right) e_{0}=64 \mu_{0} \mu_{1} \mu_{2} e\left(\chi_{3}\right) e_{0} .
$$

Together we get

$$
\mu^{2}=\mu_{0} \mu_{1} \mu_{2} .
$$

Thus $\frac{\mu}{\mu_{0}^{2}} \frac{\mu}{\mu_{1}^{2}}=\frac{\mu_{2}}{\mu_{0} \mu_{1}} \in 1+J(\mathcal{O})$. Similarly, $\frac{\mu_{1}}{\mu_{0} \mu_{2}}, \frac{\mu_{0}}{\mu_{1} \mu_{2}} \in 1+J(\mathcal{O})$. But then also $\frac{\mu_{1} \mu_{2}}{\mu_{0}} \frac{\mu_{1}}{\mu_{0} \mu_{2}}=\frac{\mu_{1}^{2}}{\mu_{0}^{2}} \in 1+J(\mathcal{O})$. Since $2 \in J(\mathcal{O})$ this implies that $\frac{\mu_{1}}{\mu_{0}} \in 1+J(\mathcal{O})$. But then actually $\mu_{2}=\frac{\mu_{1} \mu_{2}}{\mu_{0}} \frac{\mu_{0}}{\mu_{1}} \in 1+J(\mathcal{O})$. Similarly, $\mu_{0}, \mu_{1} \in 1+J(\mathcal{O})$. So we can replace $\beta$ by $\mu_{0}^{-1} \beta$, or equivalently, we can assume that $\mu_{0}=1$. Similarly, we can assume that $\mu_{1}=\mu_{2}=1$. Then $\mu^{2}=1$. If $\mu=-1$ we multiply all generators by -1 ; since $2 \in J(\mathcal{O})$, this does not change the relations over $k$, but it does change the sign of any of the above expressions $\beta \delta \lambda$ etc. involving three generators. Therefore, we can also assume that $\mu=1$.

We can now prove Theorem $\mathrm{C}$ from the introduction.

Proof of Theorem C. We assume a choice of generators of $A$ fulfilling Proposition 2.8. We show that $A$ satisfies the relations given in Theorem $\mathrm{C}$. Those in the first three lines are obvious. Since the set $\{\kappa, \kappa \lambda \kappa\}$ is an $\mathcal{O}$-basis of $e_{0} A e_{2}$, we can write

$$
\beta \delta=a \kappa+b \kappa \lambda \kappa
$$

for some unique scalars $a, b \in \mathcal{O}$. Multiplying this by $\lambda$ yields

$$
8 e\left(\chi_{3}\right) e_{0}=\beta \delta \lambda=a \kappa \lambda+b(\kappa \lambda)^{2}=(4 a+16 b) e\left(\chi_{3}\right) e_{0}+(2 a+4 b) e\left(\chi_{5}\right) e_{0} .
$$


By comparing the coefficients at $e\left(\chi_{3}\right) e_{0}$ and $e\left(\chi_{5}\right) e_{0}$ of the left and right expression in this equality, we get the equations

$$
\begin{aligned}
& 8=4 a+16 b \\
& 0=2 a+4 b .
\end{aligned}
$$

Thus the coefficients $a, b$ have values

$$
a=-2, b=1,
$$

and from this we get the following relation in the statement of Theorem $\mathrm{C}$ :

$$
\beta \delta=-2 \kappa+\kappa \lambda \kappa .
$$

In exactly the same way we get the following five relations in the Theorem:

$$
\begin{aligned}
\eta \gamma & =-2 \lambda+\lambda \kappa \lambda, \\
\delta \lambda & =-2 \gamma+\gamma \beta \gamma, \\
\kappa \eta & =-2 \beta+\beta \gamma \beta, \\
\lambda \beta & =-2 \eta+\eta \delta \eta, \\
\gamma \kappa & =-2 \delta+\delta \eta \delta .
\end{aligned}
$$

A similar technique is going to yield the remaining relations: write $\gamma \beta \delta=c \delta+d \delta \eta \delta$ for some unique $c, d \in \mathcal{O}$; as before, this is possible since $\{\delta, \delta \eta \delta\}$ is an $\mathcal{O}$-basis of $e_{1} A e_{2}$. Multiplying by $\eta$ yields

$$
\gamma \beta \delta \eta=c \delta \eta+d(\delta \eta)^{2}=c z_{2} e_{1}+d z_{2}^{2} e_{1} .
$$

The left side is equal to $(\gamma \beta)(\delta \eta)=z_{0} z_{2} e_{1}$, so comparing coefficients yields now

$$
\begin{aligned}
16 & =4 c+16 d, \\
0 & =2 c+4 d,
\end{aligned}
$$

and this implies $c=-4$ and $d=2$. Thus we get indeed

$$
\gamma \beta \delta=-4 \delta+2 \delta \eta \delta
$$

as claimed. The remaining relations of this type follow in exactly the same way.

Now consider the last three relations. Write $\beta \delta \lambda \beta=r \beta+s \beta \gamma \beta$, for $r, s \in \mathcal{O}$. Then $\beta \delta \lambda \beta \gamma=r \beta \gamma+s \beta \gamma \beta \gamma$. So

$$
32 e\left(\chi_{3}\right) e_{0}=(4 r+16 s) e\left(\chi_{3}\right) e_{0}+(2 r+4 s) e\left(\chi_{4}\right) e_{0}
$$

which yields $s=4$ and $r=-8$. The remaining two relations follow in exactly the same way. Thus $A$ satisfies all relations given in Theorem $\mathrm{C}$.

Let $\tilde{A}$ be the $\mathcal{O}$-algebra described by the generators and relations given in Theorem C. There is a surjective algebra morphism from $\tilde{A}$ to $A$. In order to show that $\tilde{A}$ and $A$ are isomorphic it suffices therefore to show that the cardinality of a minimal generating set for $\tilde{A}$ as an $\mathcal{O}$-module is at most 24 . Thus it suffices to check that the set

$$
\begin{aligned}
\mathcal{S}:= & \left\{e_{0}, e_{1}, e_{2}, \beta, \gamma, \delta, \eta, \lambda, \kappa, \beta \gamma, \gamma \beta, \delta \eta, \eta \delta, \lambda \kappa, \kappa \lambda,\right. \\
& \beta \gamma \beta, \gamma \beta \gamma, \delta \eta \delta, \eta \delta \eta, \lambda \kappa \lambda, \kappa \lambda \kappa, \beta \delta \lambda, \delta \lambda \beta, \lambda \beta \delta\}
\end{aligned}
$$


spans $\tilde{A}$ as $\mathcal{O}$-module. This is an easy consequence of the given relations; we give some details for the convenience of the reader. Let

$$
\mathcal{G}=\left\{e_{0}, e_{1}, e_{2}, \beta, \gamma, \delta, \eta, \lambda, \kappa\right\}
$$

From the given relations it is immediate that for any two elements $x, y$ of $\mathcal{G}$, $x y$ is in the $\mathcal{O}$-span of $\mathcal{S}$. Thus it suffices to show that for any two elements $x, y$ of $\mathcal{G}-\left\{e_{0}, e_{1}, e_{2}\right\}$ and any element $u$ of $\mathcal{S}-\left\{e_{0}, e_{1}, e_{2}, \beta, \gamma, \delta, \eta, \lambda, \kappa\right\}, x u$ and $u y$ are in the $\mathcal{O}$-span of $\mathcal{S}$. From the given relations we may also assume that $u$ is one of $\beta \gamma \beta, \gamma \beta \gamma, \delta \eta \delta, \eta \delta \eta, \lambda \kappa \lambda, \kappa \lambda \kappa$ or one of $\beta \delta \lambda, \delta \lambda \beta, \lambda \beta \delta$.

First, note that the relations $\kappa \eta=-2 \beta+\beta \gamma \beta$ and $\delta \lambda=-2 \gamma+\gamma \beta \gamma$ give that $\kappa \eta \gamma=\beta \delta \lambda$. Similarly, we get $\eta \gamma \kappa=\lambda \beta \delta$ and $\gamma \kappa \eta=\delta \lambda \beta$.

Now suppose $u=\beta \gamma \beta$. Then we may assume that $x$ is one of $\gamma$ or $\lambda$ and that $y$ is one of $\gamma$ or $\delta$. The relation $\kappa \eta=-2 \beta+\beta \gamma \beta$ gives $\gamma \kappa \eta=-2 \gamma \beta+\gamma \beta \gamma \beta$, hence $\gamma \beta \gamma \beta$ is in the $\mathcal{O}$-span of $\mathcal{S}$. The relation $\kappa \eta=-2 \beta+\beta \gamma \beta$ also gives $\lambda \kappa \eta=-2 \lambda \beta+\lambda \beta \gamma \beta$. It follows from the relation $\lambda \kappa \eta=-4 \eta+2 \eta \delta \eta$ that $\lambda \beta \gamma \beta$ is in the $\mathcal{O}$-span of $\mathcal{S}$. We show similarly that $\beta \gamma \beta \gamma$ and $\beta \gamma \beta \delta$ are in the $\mathcal{O}$-span of $\mathcal{S}$.

The cases $u=\gamma \beta \gamma, \delta \eta \delta, \eta \delta \eta, \lambda \kappa \lambda, \kappa \lambda \kappa$ are handled analogously.

Now suppose $u=\beta \delta \lambda$. Then we may assume that $x$ is one of $\lambda$ or $\gamma$ and $y$ is one of $\beta$ or $\kappa$. The relation $\lambda \beta \delta \lambda=-8 \lambda+4 \lambda \kappa \lambda$ shows that $\lambda \beta \delta \lambda$ is in the $\mathcal{O}$-span of $\mathcal{S}$. From the relation $\gamma \beta \delta=-4 \delta+2 \delta \eta \delta$ we get $\gamma \beta \delta \lambda=-4 \delta \lambda+2 \delta \eta \delta \lambda$. From $\gamma \kappa=-2 \delta+\delta \eta \delta$, we get $\delta \eta \delta \lambda=\gamma \kappa \lambda+2 \delta \lambda$. Hence $\delta \eta \delta \lambda$ is in the $\mathcal{O}$-span of $\mathcal{S}$, and so is $\gamma \beta \delta \lambda$. We argue similarly to show that $\beta \delta \lambda \beta$ and $\beta \delta \lambda \kappa$ are in the $\mathcal{O}$-span of $\mathcal{S}$.

The cases $u=\delta \lambda \beta$ and $u=\lambda \beta \delta$ are handled in the same fashion.

REMARK 2.9. An interesting consequence of 2.5 is the structure of $e A e$ for any primitive idempotent $e$ in $A$. We have an $\mathcal{O}$-algebra isomorphism

$$
e A e \cong \mathcal{O}[X, Y] /\left\langle X^{2}-Y^{2}-2(X-Y), X Y-2 X^{2}+4 X\right\rangle
$$

indeed, we may assume that $e=e_{0}$, and then the assignment $X \mapsto z_{0} e_{0}, Y \mapsto z_{1} e_{0}$ induces the required isomorphism. In particular, we have an isomorphism of $k$-algebras

$$
\bar{e} \bar{A} \bar{e} \cong k[X, Y] /\left\langle X^{2}-Y^{2}, X Y\right\rangle .
$$

This is, by Erdmann [6, III.1, III.3], up to isomorphism the unique 4-dimensional symmetric $k$-algebra which is not isomorphic to the group algebra of the Klein four group. One might be tempted to ask whether any symmetric $\mathcal{O}$-algebra is the endomorphism algebra of some projective module of some block algebra.

\section{REFERENCES}

1. M. Broué, Isométries parfaites, types de blocs, catégories dérivées, Astérisque 181-182 (1990), 61-92.

2. M. Broué, Isométries de caractères et equivalences de Morita ou dérivées, Publ. Math. IHES 71 (1990), 45-63. 128.

3. M. Broué and L. Puig, A Frobenius theorem for blocks, Invent. Math. 56 (1980), 117

4. M. Cabanes and C. Picaronny, Types of blocks with dihedral or quaternion defect groups, J. Fac. Sci. Univ. Tokyo 39 (1992), 141-161. 
5. M. Cabanes and C. Picaronny, Corrected version of: Types of blocks with dihedral or quaternion defect groups, http://www.math. jussieu.fr/〜cabanes/printlist.html (1999).

6. K. Erdmann, Blocks of tame representation type and related algebras, Lecture Notes in Mathematics No. 1428 (Springer-Verlag, 1990).

7. W. Feit, The representation theory of finite groups (North-Holland, Amsterdam, 1982).

8. L. Puig, Nilpotent blocks and their source algebras, Invent. Math. 93 (1988), 77-116. 1995).

9. J. Thévenaz, G-algebras and modular representation theory (Oxford University Press, 